Article

\title{
Positive Solutions for a System of Fractional Integral Boundary Value Problems of Riemann-Liouville Type Involving Semipositone Nonlinearities
}

\author{
Youzheng Ding ${ }^{1}$, Jiafa $\mathrm{Xu}^{2}$ and Zhengqing $\mathrm{Fu}^{3, *}$ \\ 1 School of Science, Shandong Jianzhu University, Jinan 250101, China; dingyz@sdjzu.edu.cn \\ 2 School of Mathematical Sciences, Chongqing Normal University, Chongqing 401331, China; \\ 20150028@cqnu.edu.cn \\ 3 College of Mathematics and System Sciences, Shandong University of Science and Technology, \\ Qingdao 266590, China \\ * Correspondence: skd992050@sdust.edu.cn
}

Received: 18 September 2019; Accepted: 8 October 2019; Published: 14 October 2019

Abstract: In this work by the index of fixed point and matrix theory, we discuss the positive solutions for the system of Riemann-Liouville type fractional boundary value problems

$$
\begin{gathered}
D_{0+}^{\alpha} u(t)+f_{1}(t, u(t), v(t), w(t))=0, t \in(0,1), \\
D_{0+}^{\alpha} v(t)+f_{2}(t, u(t), v(t), w(t))=0, t \in(0,1), \\
D_{0+}^{\alpha} w(t)+f_{3}(t, u(t), v(t), w(t))=0, t \in(0,1), \\
u(0)=u^{\prime}(0)=\cdots=u^{(n-2)}(0)=0,\left.D_{0+}^{p} u(t)\right|_{t=1}=\int_{0}^{1} h(t) D_{0+}^{q} u(t) d t, \\
v(0)=v^{\prime}(0)=\cdots=v^{(n-2)}(0)=0,\left.D_{0+}^{p} v(t)\right|_{t=1}=\int_{0}^{1} h(t) D_{0+}^{q} v(t) d t, \\
w(0)=w^{\prime}(0)=\cdots=w^{(n-2)}(0)=0,\left.D_{0+}^{p} w(t)\right|_{t=1}=\int_{0}^{1} h(t) D_{0+}^{q} w(t) d t,
\end{gathered}
$$

where $\alpha \in(n-1, n]$ with $n \in \mathbb{N}, n \geq 3, p, q \in \mathbb{R}$ with $p \in[1, n-2], q \in[0, p], D_{0+}^{\alpha}$ is the $\alpha$ order Riemann-Liouville type fractional derivative, and $f_{i}(i=1,2,3) \in C\left([0,1] \times \mathbb{R}^{+} \times \mathbb{R}^{+} \times \mathbb{R}^{+}, \mathbb{R}\right)$ are semipositone nonlinearities.

Keywords: Riemann-Liouville type fractional problem; positive solutions; the index of fixed point; matrix theory

\section{Introduction}

In this work the positive solutions for the system of fractional boundary value problems involving Riemann-Liouville type are considered:

$$
\left\{\begin{array}{l}
D_{0+}^{\alpha} u(t)+f_{1}(t, u(t), v(t), w(t))=0, t \in(0,1) \\
D_{0+}^{\alpha} v(t)+f_{2}(t, u(t), v(t), w(t))=0, t \in(0,1) \\
D_{0+}^{\alpha} w(t)+f_{3}(t, u(t), v(t), w(t))=0, t \in(0,1) \\
u(0)=u^{\prime}(0)=\cdots=u^{(n-2)}(0)=0,\left.D_{0+}^{p} u(t)\right|_{t=1}=\int_{0}^{1} h(t) D_{0+}^{q} u(t) d t \\
v(0)=v^{\prime}(0)=\cdots=v^{(n-2)}(0)=0,\left.D_{0+}^{p} v(t)\right|_{t=1}=\int_{0}^{1} h(t) D_{0+}^{q} v(t) d t \\
w(0)=w^{\prime}(0)=\cdots=w^{(n-2)}(0)=0,\left.D_{0+}^{p} w(t)\right|_{t=1}=\int_{0}^{1} h(t) D_{0+}^{q} w(t) d t,
\end{array}\right.
$$


where $D_{0+}^{\alpha}$ is the $\alpha$ order Riemann-Liouville type fractional derivative, the constants $\alpha, p, q, n$, and the functions $h, f_{i}(i=1,2,3)$ satisfy the assumptions

(C0) $n \in \mathbb{N}, n \geq 3, \alpha \in(n-1, n], p \in[1, n-2], q \in[0, p]$,

(C1) there exists $h$ with $h(t) \geq 0(\not \equiv 0)$ on $[0,1]$ such that $A:=\frac{\Gamma(\alpha)}{\Gamma(\alpha-p)}-\frac{\Gamma(\alpha)}{\Gamma(\alpha-q)} \int_{0}^{1} h(t) t^{\alpha-q-1} d t>0$,

(C2) $f_{i}(i=1,2,3) \in C\left([0,1] \times \mathbb{R}^{+} \times \mathbb{R}^{+} \times \mathbb{R}^{+}, \mathbb{R}\right)$, and there is a $M>0$ such that

$$
f_{i}\left(t, x_{1}, x_{2}, x_{3}\right) \geq-M, \text { for }\left(t, x_{1}, x_{2}, x_{3}\right) \in[0,1] \times \mathbb{R}^{+} \times \mathbb{R}^{+} \times \mathbb{R}^{+}, i=1,2,3 .
$$

Fractional calculus theory shows undoubted advantages in aerodynamics, electrodynamics in complex medium, the theory of control, signal and image processing, rheology, and many other issues, see the books [1-3]. The study of such kind of problems has received considerable attention in the previous studies, see for instance [4-79] and the references therein.

In [4] by the fixed point theorem of Guo-Krasnosel'skii, the authors discussed the positive solutions for the multi-point Riemann-Liouville fractional boundary value problems

$$
\left\{\begin{array}{l}
D_{0+}^{\alpha} u(t)+\lambda f(t, u(t))=0, t \in(0,1), \\
u(0)=u^{\prime}(0)=\cdots=u^{(n-2)}(0)=0, \\
\left.D_{0+}^{p} u(t)\right|_{t=1}=\left.\sum_{i=1}^{m} a_{i} D_{0+}^{q} u(t)\right|_{t=\xi_{i}}
\end{array}\right.
$$

where $f$ is a sign-changing nonlinearity. In [5], the authors studied the multiple positive solutions for the problem (2) $(\lambda=1)$, where $f$ is a sign-changing nonlinearity, and permits singularities on $t$ and $u$. In [6], by means of the index of fixed point, the authors researched the positive solutions for the boundary value problems of Hadamard fractional equations

$$
\left\{\begin{array}{l}
-{ }^{H} D^{\alpha} u(t)=f(t, u(t)), \quad t \in[1, e], \\
u(1)=\delta u(1)=\delta u(e)=0,
\end{array}\right.
$$

where $f$ is a sign-changing nonlinearity, and may grow superlinearly and sublinearly at $\infty$.

The fractional-order equations in systems have also been widely investigated in the literature, see for example [52-79]. In [52], the authors studied the system of Hadamard fractional integral boundary value problems

$$
\left\{\begin{array}{l}
{ }^{H} D^{\beta} u(t)+f_{1}(t, u(t), v(t))=0, \quad 1<t<e, \\
{ }^{H} D^{\beta} v(t)+f_{2}(t, u(t), v(t))=0, \quad 1<t<e, \\
u(1)=v(1)=u^{\prime}(1)=v^{\prime}(1)=0, \\
u(e)=\int_{1}^{e} h(s) v(s) \frac{d s}{s} \\
v(e)=\int_{1}^{e} g(s) u(s) \frac{d s}{s},
\end{array}\right.
$$

where the nonlinearities $f_{i}(i=1,2) \in C\left([1, e] \times \mathbb{R}^{+} \times \mathbb{R}^{+}, \mathbb{R}^{+}\right)$.

In [53], by means of the alternative of Leray-Schauder, the authors obtained the uniqueness and existence of solutions for the system of fractional integral boundary value problems

$$
\left\{\begin{array}{l}
D^{\alpha} x(t)=f\left(t, x(t), y(t), D^{\gamma} y(t)\right), t \in[0, T], \\
D^{\beta} y(t)=g\left(t, x(t), D^{\delta} x(t), y(t)\right), t \in[0, T],
\end{array}\right.
$$

with the integral boundary conditions

$$
\left\{\begin{array}{l}
x(0)=h(y), \int_{0}^{T} y(s) d s=\mu_{1} x(\eta) \\
y(0)=\phi(x), \int_{0}^{T} x(s) d s=\mu_{2} y(\xi)
\end{array}\right.
$$

where $D^{\alpha}, D^{\beta}, D^{\delta}, D^{\gamma}$ are the fractional derivatives of Caputo type. 
In [54], the authors studied the positive solutions of the abstract fractional semipositone differential system with integral boundary conditions, which arises from HIV infection models

$$
\left\{\begin{array}{l}
D_{0+}^{\alpha} u(t)+\lambda f\left(t, u(t), D_{0+}^{\beta} u(t), v(t)\right)=0, \\
D_{0+}^{\gamma} v(t)+\lambda g(t, u(t))=0,0<t<1, \\
D_{0+}^{\beta} u(0)=D_{0+}^{\beta+1} u(0)=0, D_{0+}^{\beta} u(1)=\int_{0}^{1} D_{0+}^{\beta} u(s) d A(s), \\
v(0)=v^{\prime}(0)=0, v(1)=\int_{0}^{1} v(s) d B(s),
\end{array}\right.
$$

where $f, g$ are the semipositone nonlinearities (so-called semipositone problems), which originally modeled nonlinear phenomena of chemical reactions by Dutch chemist Aris [80]. For some relevant work, we refer the reader to $[4-7,71-75]$.

Motivated by the works aforementioned, in this work we use the index of fixed point and nonnegative matrix theory to study the positive solutions for the system of Riemann-Liouville type fractional boundary value problems (1). We first transform our problem into the equivalent system of Hammerstein type integral equations, and establish some nonnegative operator equations. Then, using some superlinear and sublinear conditions for our nonlinearities, we obtain two existence theorems. Finally, we offer two examples to explain our main theorems.

\section{Preliminaries}

Now, we offer the definition of the $\alpha(>0)$ order Riemann-Liouville type fractional derivative, which is given by

$$
D_{0+}^{\alpha} f(t)=\frac{1}{\Gamma(n-\alpha)}\left(\frac{\mathrm{d}}{\mathrm{d} t}\right)^{n} \int_{0}^{t}(t-s)^{n-\alpha-1} f(s) d s,
$$

where $f:(0,+\infty) \rightarrow(-\infty,+\infty)$ is a continuous function, and $n=[\alpha]+1$. For more materials, we refer to the books [1-3].

Lemma 1. ISuppose that (C0)-(C1) hold. Let $f \in C[0,1]$, then the problem

$$
\left\{\begin{array}{l}
D_{0+}^{\alpha} u(t)+f(t)=0, t \in(0,1) \\
u(0)=u^{\prime}(0)=\cdots=u^{(n-2)}(0)=0,\left.D_{0+}^{p} u(t)\right|_{t=1}=\int_{0}^{1} h(t) D_{0+}^{q} u(t) d t
\end{array}\right.
$$

has a solution, which can take the form

$$
u(t)=\int_{0}^{1} G(t, s) f(s) d s,
$$

where

$$
G(t, s)=g_{1}(t, s)+\frac{t^{\alpha-1}}{A} \int_{0}^{1} h(t) g_{2}(t, s) d t
$$

and

$$
\begin{gathered}
g_{1}(t, s)=\frac{1}{\Gamma(\alpha)} \begin{cases}t^{\alpha-1}(1-s)^{\alpha-p-1}-(t-s)^{\alpha-1}, & 0 \leq s \leq t \leq 1, \\
t^{\alpha-1}(1-s)^{\alpha-p-1}, & 0 \leq t \leq s \leq 1,\end{cases} \\
g_{2}(t, s)=\frac{1}{\Gamma(\alpha-q)} \begin{cases}t^{\alpha-q-1}(1-s)^{\alpha-p-1}-(t-s)^{\alpha-q-1}, & 0 \leq s \leq t \leq 1, \\
t^{\alpha-q-1}(1-s)^{\alpha-p-1}, & 0 \leq t \leq s \leq 1 .\end{cases}
\end{gathered}
$$

Proof. Using similar arguments in ([4], [Lemma 1 and 2]), we have

$$
u(t)=c_{1} t^{\alpha-1}+c_{2} t^{\alpha-2}+\cdots+c_{n} t^{\alpha-n}-\int_{0}^{t} \frac{(t-s)^{\alpha-1}}{\Gamma(\alpha)} f(s) d s,
$$


where $c_{i} \in \mathbb{R}, i=1,2, \ldots, n$. Note that $u(0)=u^{\prime}(0)=\cdots=u^{(n-2)}(0)=0$, and thus $c_{2}=\cdots=c_{n}=0$. Consequently, we get

$$
u(t)=c_{1} t^{\alpha-1}-\int_{0}^{t} \frac{(t-s)^{\alpha-1}}{\Gamma(\alpha)} f(s) d s
$$

Therefore, we find

$$
D_{0+}^{p} u(t)=c_{1} \frac{\Gamma(\alpha)}{\Gamma(\alpha-p)} t^{\alpha-p-1}-I_{0+}^{\alpha-p} f(t), D_{0+}^{q} u(t)=c_{1} \frac{\Gamma(\alpha)}{\Gamma(\alpha-q)} t^{\alpha-q-1}-I_{0+}^{\alpha-q} f(t) .
$$

Using the condition $\left.D_{0+}^{p} u(t)\right|_{t=1}=\int_{0}^{1} h(t) D_{0+}^{q} u(t) d t$, we have

$$
c_{1} \frac{\Gamma(\alpha)}{\Gamma(\alpha-p)}-\frac{1}{\Gamma(\alpha-p)} \int_{0}^{1}(1-s)^{\alpha-p-1} f(s) d s=c_{1} \frac{\Gamma(\alpha)}{\Gamma(\alpha-q)} \int_{0}^{1} h(t) t^{\alpha-q-1} d t-\frac{1}{\Gamma(\alpha-q)} \int_{0}^{1} h(t) \int_{0}^{t}(t-s)^{\alpha-q-1} f(s) d s d t .
$$

Solving this equation, we obtain

$$
c_{1}=\frac{1}{A \Gamma(\alpha-p)} \int_{0}^{1}(1-s)^{\alpha-p-1} f(s) d s-\frac{1}{A \Gamma(\alpha-q)} \int_{0}^{1} h(t) \int_{0}^{t}(t-s)^{\alpha-q-1} f(s) d s d t .
$$

As a result, we get

$$
\begin{aligned}
u(t)= & \frac{1}{A \Gamma(\alpha-p)} \int_{0}^{1} t^{\alpha-1}(1-s)^{\alpha-p-1} f(s) d s-\frac{t^{\alpha-1}}{A \Gamma(\alpha-q)} \int_{0}^{1} h(t) \int_{0}^{t}(t-s)^{\alpha-q-1} f(s) d s d t-\int_{0}^{t} \frac{(t-s)^{\alpha-1}}{\Gamma(\alpha)} f(s) d s \\
= & \frac{1}{\Gamma(\alpha)} \int_{0}^{1} t^{\alpha-1}(1-s)^{\alpha-p-1} f(s) d s-\int_{0}^{t} \frac{(t-s)^{\alpha-1}}{\Gamma(\alpha)} f(s) d s+\left[\frac{1}{A \Gamma(\alpha-p)}-\frac{1}{\Gamma(\alpha)}\right] \int_{0}^{1} t^{\alpha-1}(1-s)^{\alpha-p-1} f(s) d s \\
& -\frac{t^{\alpha-1}}{A \Gamma(\alpha-q)} \int_{0}^{1} h(t) \int_{0}^{t}(t-s)^{\alpha-q-1} f(s) d s d t \\
= & \int_{0}^{1} g_{1}(t, s) f(s) d s+\frac{t^{\alpha-1}}{A \Gamma(\alpha-q)}\left[\int_{0}^{1} \int_{0}^{1} h(t) t^{\alpha-q-1}(1-s)^{\alpha-p-1} f(s) d s d t-\int_{0}^{1} h(t) \int_{0}^{t}(t-s)^{\alpha-q-1} f(s) d s d t\right] \\
= & \int_{0}^{1} g_{1}(t, s) f(s) d s+\frac{t^{\alpha-1}}{A} \int_{0}^{1} \int_{0}^{1} h(t) g_{2}(t, s) d t f(s) d s \\
= & \int_{0}^{1} G(t, s) f(s) d s .
\end{aligned}
$$

Lemma 2. (see ([4], [Lemma 3])). Suppose that (C0) holds. The functions $g_{i}(i=1,2)$ have the properties (i) $g_{i} \in C\left([0,1] \times[0,1], \mathbb{R}^{+}\right)$, and $g_{i}(t, s)>0$ for $t, s \in(0,1), i=1,2$,

(ii) $t^{\alpha-1} \widetilde{\varphi}(s) \leq g_{1}(t, s) \leq \widetilde{\varphi}(s)$ for all $t, s \in[0,1]$, where

$$
\widetilde{\varphi}(s)=\frac{(1-s)^{\alpha-p-1}\left(1-(1-s)^{p}\right)}{\Gamma(\alpha)}, s \in[0,1],
$$

(iii) $g_{1}(t, s) \leq \frac{t^{\alpha-1}(1-s)^{\alpha-p-1}}{\Gamma(\alpha)}, t, s \in[0,1]$.

Lemma 3. Suppose that (CO)-(C1) hold. The Green's function $G$ has the properties

(i) $G \in C\left([0,1] \times[0,1], \mathbb{R}^{+}\right)$, and $G(t, s)>0$ for $t, s \in(0,1)$,

(ii) $t^{\alpha-1} \varphi(s) \leq G(t, s) \leq \varphi(s), \forall t, s \in[0,1]$, where

$$
\varphi(s)=\widetilde{\varphi}(s)+\frac{1}{A} \int_{0}^{1} h(t) g_{2}(t, s) d t, s \in[0,1],
$$

(iii) $G(t, s) \leq t^{\alpha-1}\left[\frac{(1-s)^{\alpha-p-1}}{\Gamma(\alpha)}+\frac{1}{A} \int_{0}^{1} h(t) g_{2}(t, s) d t\right], \forall t, s \in[0,1]$.

This is a direct result of Lemma 2, so we omit its proof. 
Lemma 4. Let $\kappa_{1}=\int_{0}^{1} t^{\alpha-1} \varphi(t) d t, \kappa_{2}=\int_{0}^{1} \varphi(t) d t$. Then we have the following inequalities

$$
\kappa_{1} \varphi(s) \leq \int_{0}^{1} G(t, s) \varphi(t) d t \leq \kappa_{2} \varphi(s), \forall s \in[0,1]
$$

From Lemma 3(ii), we easily obtain (10).

Next we will consider the problem

$$
\left\{\begin{array}{l}
D_{0+}^{\alpha} u(t)+\widetilde{f}(t, u(t))=0, t \in(0,1) \\
u(0)=u^{\prime}(0)=\cdots=u^{(n-2)}(0)=0,\left.D_{0+}^{p} u(t)\right|_{t=1}=\int_{0}^{1} h(t) D_{0+}^{q} u(t) d t
\end{array}\right.
$$

where $\widetilde{f}$ satisfies the condition

$(\mathrm{C} 2)^{\prime} \tilde{f} \in C\left([0,1] \times \mathbb{R}^{+}, \mathbb{R}\right)$, and there is a $M>0$ such that

$$
\widetilde{f}\left(t, x_{1}\right) \geq-M, \text { for }\left(t, x_{1}\right) \in[0,1] \times \mathbb{R}^{+} .
$$

Lemma 5. Suppose that $(\mathrm{C} 0)-(\mathrm{C} 1)$ and $(\mathrm{C} 2)^{\prime}$. Then the problem (11) is equivalent to

$$
u(t)=\int_{0}^{1} G(t, s) \widetilde{f}(s, u(s)) d s,
$$

where $G$ is defined in Lemma 1.

Now, we take care of the following auxiliary problem associated with (11):

$$
\left\{\begin{array}{l}
D_{0+}^{\alpha} u(t)+\widetilde{F}(t, u(t)-z(t))=0, t \in(0,1) \\
u(0)=u^{\prime}(0)=\cdots=u^{(n-2)}(0)=0,\left.D_{0+}^{p} u(t)\right|_{t=1}=\int_{0}^{1} h(t) D_{0+}^{q} u(t) d t
\end{array}\right.
$$

where $\widetilde{F}\left(t, x_{1}\right)=\left\{\begin{array}{ll}\widetilde{f}\left(t, x_{1}\right)+M, & t \in[0,1], x_{1} \geq 0, \\ \widetilde{f}(t, 0)+M, & t \in[0,1], x_{1}<0,\end{array}\right.$ and $z(t)=M \int_{0}^{1} G(t, s) d s$, for $t \in[0,1]$. Then $\widetilde{F}$ is nonnegative continuous on $[0,1] \times \mathbb{R}^{+}$, and from Lemma 5 we have (13) is equivalent to

$$
u(t)=\int_{0}^{1} G(t, s) \widetilde{F}(s, u(s)-z(s)) d s,
$$

where $G$ is as in Lemma 1.

Lemma 6. (i) If (11) has a positive solution $u^{*}$, then (13) has a solution $u^{*}+z$.

(ii) If $u^{*}$ is a solution for (13), and $u^{*}(t) \geq z(t)$ for $t \in[0,1]$, then $u^{*}-z$ is a positive solution for (11).

Proof. Note that $z$ satisfies the fractional boundary value problem

$$
\left\{\begin{array}{l}
D_{0+}^{\alpha} z(t)+M=0, t \in(0,1) \\
z(0)=z^{\prime}(0)=\cdots=z^{(n-2)}(0)=0,\left.D_{0+}^{p} z(t)\right|_{t=1}=\int_{0}^{1} h(t) D_{0+}^{q} z(t) d t
\end{array}\right.
$$

Substituting $u^{*}+z$ into (13), we have

$$
D_{0+}^{\alpha}\left(u^{*}+z\right)(t)+\widetilde{F}\left(t, u^{*}(t)+z(t)-z(t)\right)=0 \Longrightarrow D_{0+}^{\alpha} u^{*}(t)+D_{0+}^{\alpha}(z)(t)+\widetilde{f}\left(t, u^{*}(t)\right)+M=0 .
$$

Using $D_{0+}^{\alpha}(z)(t)=-M$, we have $D_{0+}^{\alpha} u^{*}(t)+\widetilde{f}\left(t, u^{*}(t)\right)=0$, and note that $u^{*}, z$ satisfy the boundary conditions in (11), (15), we obtain Lemma 6(i) holds.

Next, substituting $u^{*}-z$ into (11), and using $D_{0+}^{\alpha}(z)(t)=-M$ we have

$$
D_{0+}^{\alpha}\left(u^{*}-z\right)(t)+\widetilde{f}\left(t, u^{*}(t)-z(t)\right)=0 \Longrightarrow D_{0+}^{\alpha} u^{*}(t)-D_{0+}^{\alpha} z(t)+\widetilde{f}\left(t, u^{*}(t)-z(t)\right)=0,
$$


and

$$
D_{0+}^{\alpha} u^{*}(t)+\widetilde{F}\left(t, u^{*}(t)-z(t)\right)=0 .
$$

Note that $u^{*}, z$ satisfy the boundary conditions in (13), (15), we obtain Lemma 6(ii) holds.

Lemma 6 implies that we only need to seek the solution $u^{*}$ for (13), which is greater than $z$, we can obtain the positive solution $u^{*}-z$ for (11).

Let $E:=C[0,1],\|u\|:=\max _{t \in[0,1]}|u(t)|, P:=\{u \in E: u(t) \geq 0, \forall t \in[0,1]\}, P_{0}=\{u \in P: u(t) \geq$ $\left.t^{\alpha-1}\|u\|, \forall t \in[0,1]\right\}$. Then $(E,\|\cdot\|)$ is a real Banach space, and $P, P_{0}$ are cones on $E$. Note that the relations between (13) and (14), we let an operator $T: P \rightarrow P$ as follows:

$$
(T u)(t)=\int_{0}^{1} G(t, s) \widetilde{F}(s, u(s)-z(s)) d s, \text { for } u \in P, t \in[0,1] .
$$

From the continuity of $G, \widetilde{F}$ we obtain $T: P \rightarrow P$ is a completely continuous operator, and if there exists $\bar{u} \in P \backslash\{0\}$ such that $T \bar{u}=\bar{u}$, then this $\bar{u}$ is a positive solution for (13).

Lemma 7. $T(P) \subset P_{0}$.

By Lemma 3(ii) we can easily obtain this conclusion, so we omit its proof.

Note that if $\bar{u}$ is a positive fixed point of $T$, from Lemma 7 we have $\bar{u} \in P_{0}$. Moreover, when

$$
\|\bar{u}\| \geq \widetilde{M}=M \int_{0}^{1}\left[\frac{(1-s)^{\alpha-p-1}}{\Gamma(\alpha)}+\frac{1}{A} \int_{0}^{1} h(t) g_{2}(t, s) d t\right] d s>0,
$$

we have

$$
\begin{aligned}
\bar{u}(t)-z(t) & \geq t^{\alpha-1}\|\bar{u}\|-M \int_{0}^{1} G(t, s) d s \\
& \geq t^{\alpha-1}\|\bar{u}\|-M \int_{0}^{1} t^{\alpha-1}\left[\frac{(1-s)^{\alpha-p-1}}{\Gamma(\alpha)}+\frac{1}{A} \int_{0}^{1} h(t) g_{2}(t, s) d t\right] d s \\
& \geq 0 .
\end{aligned}
$$

Then from Lemma 6 we have $\bar{u}-z$ is a positive solution for (11). Therefore, we only need to study the positive fixed point $u^{*}$ for $T$, which the norm is greater than $\widetilde{M}$, then $u^{*}-z$ is a positive solution for (11).

In the following two lemmas, we let $X$ be a real Banach space and $P$ a cone on $X$.

Lemma 8. (see [81]). Let $\Omega \subset X$ be a bounded open set, and $T: \bar{\Omega} \cap P \rightarrow P$ a continuous compact operator. If there exists $\mu_{0} \in P \backslash\{0\}$ such that

$$
u-T u \neq \lambda \mu_{0}, \forall \lambda \geq 0, u \in \partial \Omega \cap P,
$$

then $i(T, \Omega \cap P, P)=0$, where $i$ is the index of fixed point on $P$.

Lemma 9. (see [81]). Let $\Omega \subset X$ be a bounded open set with $0 \in \Omega$, and $T: \bar{\Omega} \cap P \rightarrow P$ a continuous compact operator. If

$$
u-\lambda T u \neq 0, \forall \lambda \in[0,1], u \in \partial \Omega \cap P,
$$

then $i(T, \Omega \cap P, P)=1$.

In what follows, in order to build our main theorems, we need to introduce some basic knowledge for nonnegative matrices, for more details see $[82,83]$.

Definition 1. Let $\mathcal{M}$ be a real matrix. If all elements of $\mathcal{M}$ are nonnegative, then $\mathcal{M}$ is called to be nonnegative. 
Definition 2. A real square matrix $\mathcal{M}=\left(m_{i j}\right)_{n \times n}$ is called $\mathbb{R}_{+}^{n}$-monotone, if for every column vector $x \in \mathbb{R}^{n}, \mathcal{M} x \in \mathbb{R}_{+}^{n} \Longrightarrow x \in \mathbb{R}_{+}^{n}$.

Lemma 10. A real square matrix $\mathcal{M}$ is $\mathbb{R}_{+}^{n}$-monotone $\Longleftrightarrow \operatorname{det} \mathcal{M} \neq 0$, and $\mathcal{M}^{-1}$ is nonnegative.

Remark 1. Note that our boundary condition at $t=1$ is integral and generalizes multi-point fractional boundary conditions. However, our problem (7) can be considered as a perturbation of the two-point boundary value problem

$$
\left\{\begin{array}{l}
D_{0+}^{\alpha} u(t)+f(t)=0, t \in(0,1), \\
u(0)=u^{\prime}(0)=\cdots=u^{(n-2)}(0)=\left.D_{0+}^{p} u(t)\right|_{t=1}=0,
\end{array}\right.
$$

which is equivalent to

$$
u(t)=\int_{0}^{1} g_{1}(t, s) f(s) d s,
$$

where $g_{1}$ is defined by (8). Therefore, our method, by making good use of the original Green's function for the problem (16), will dispense with constructing a new Green's function, in contrast to some papers dealing with multi-point boundary value problems. For example, in [50] the author studied the problem

$$
\left\{\begin{array}{l}
D_{0+}^{\alpha} u(t)+f(t, u(t))=0,0<t<1 \\
u(0)=0, \beta u(\eta)=u(1)
\end{array}\right.
$$

where $\alpha \in(1,2], \beta \eta^{\alpha-1}, \eta \in(0,1)$. The author obtained the Green's function associated with (17) is

$$
G_{B a i}(t, s)= \begin{cases}\frac{[t(1-s)]^{\alpha-1}-\beta t^{\alpha-1}(\eta-s)^{\alpha-1}-(t-s)^{\alpha-1}\left(1-\beta \eta^{\alpha-1}\right)}{\left(1-\beta \eta^{\alpha-1}\right) \Gamma(\alpha)}, & 0 \leq s \leq t \leq 1, s \leq \eta, \\ \frac{[t(1-s)]^{\alpha-1}-(t-s)^{\alpha-1}\left(1-\beta \eta^{\alpha-1}\right)}{\left(1-\beta \eta^{\alpha-1}\right) \Gamma(\alpha)}, & 0<\eta \leq s \leq t \leq 1, \\ \frac{[t(1-s)]^{\alpha-1}-\beta^{\alpha-1}(\eta-s)^{\alpha-1}}{\left(1-\beta \eta^{\alpha-1}\right) \Gamma(\alpha)}, & 0 \leq t \leq s \leq \eta<1, \\ \frac{[t(1-s)]^{\alpha-1}}{\left(1-\beta \eta^{\alpha-1}\right) \Gamma(\alpha)}, & 0 \leq t \leq s \leq 1, \eta \leq s .\end{cases}
$$

This function is very complicated. However, we note that this function can be expressed by

$$
\begin{aligned}
& G_{B a i}(t, s)=g_{\text {Bai }}(t, s)+\frac{\beta t^{\alpha-1}}{1-\beta \eta^{\alpha-1}} g_{\text {Bai }}(\eta, s), \\
& g_{\text {Bai }}(t, s)=\frac{1}{\Gamma(\alpha)} \begin{cases}{[t(1-s)]^{\alpha-1}-(t-s)^{\alpha-1},} & 0 \leq s \leq t \leq 1, \\
{[t(1-s)]^{\alpha-1}} & 0 \leq t \leq s \leq 1,\end{cases}
\end{aligned}
$$

where $g_{B a i}$ is the Green's function for the problem

$$
\left\{\begin{array}{l}
D_{0+}^{\alpha} u(t)+f(t, u(t))=0,0<t<1, \\
u(0)=u(1)=0 .
\end{array}\right.
$$

Compared with $G_{B a i}, g_{B a i}$ is much simpler.

\section{Main Results}

From the discission of Section 2, we can define the operators $T_{i}(i=1,2,3): P \times P \times P \rightarrow P$ and $T: P \times P \times P \rightarrow P \times P \times P$ as follows:

$$
\begin{aligned}
& T_{i}(u, v, w)(t)=\int_{0}^{1} G(t, s) F_{i}(s, u(s)-z(s), v(s)-z(s), w(s)-z(s)) d s, \\
& T(u, v, w)(t)=\left(T_{1}, T_{2}, T_{3}\right)(u, v, w)(t), \text { for } t \in[0,1],
\end{aligned}
$$


where $F_{i}\left(t, x_{1}, x_{2}, x_{3}\right)=\left\{\begin{array}{ll}f_{i}\left(t, x_{1}, x_{2}, x_{3}\right)+M, & t \in[0,1], \text { for } x_{i} \geq 0, i=1,2,3, \\ f_{i}(t, 0,0,0)+M, & t \in[0,1], \text { for else cases. }\end{array}\right.$ Consequently, if there exists $(\bar{u}, \bar{v}, \bar{w})$ is a positive fixed pint of $T$ with $\|\bar{u}\|,\|\bar{v}\|,\|\bar{w}\| \geq \widetilde{M}$, then we obtain $(\bar{u}-z, \bar{v}-z, \bar{w}-z)$ is a positive solution for (1).

Now, we list our assumptions for $F_{i}(i=1,2,3)$ :

(C3) There exist $a_{j i}, b_{j i} \geq 0$ and $l_{j}>0(i, j=1,2,3)$ such that

$$
\left(\begin{array}{l}
F_{1}\left(t, x_{1}, x_{2}, x_{3}\right) \\
F_{2}\left(t, x_{1}, x_{2}, x_{3}\right) \\
F_{3}\left(t, x_{1}, x_{2}, x_{3}\right)
\end{array}\right) \geq\left(\begin{array}{l}
a_{11} x_{1}+a_{12} x_{2}+a_{13} x_{3}-l_{1} \\
a_{21} x_{1}+a_{22} x_{2}+a_{23} x_{3}-l_{2} \\
a_{31} x_{1}+a_{32} x_{2}+a_{33} x_{3}-l_{3}
\end{array}\right), \forall\left(t, x_{1}, x_{2}, x_{3}\right) \in[0,1] \times \mathbb{R}^{+} \times \mathbb{R}^{+} \times \mathbb{R}^{+},
$$

and the matrix $\mathcal{M}_{1}$ is a $\mathbb{R}_{+}^{3}$-monotone matrix, where

$$
\mathcal{M}_{1}=\left(\begin{array}{ccc}
\kappa_{1} a_{11}-1 & \kappa_{1} a_{12} & \kappa_{1} a_{13} \\
\kappa_{1} a_{21} & \kappa_{1} a_{22}-1 & \kappa_{1} a_{23} \\
\kappa_{1} a_{31} & \kappa_{1} a_{32} & \kappa_{1} a_{33}-1
\end{array}\right)
$$

(C4) There exists $Q_{i}(t)$ in $[0,1]$ such that

$\int_{0}^{1} \varphi(t) Q_{i}(t) d t<\widetilde{M}$, and $F_{i}\left(t, x_{1}, x_{2}, x_{3}\right) \leq Q_{i}(t), \forall\left(t, x_{1}, x_{2}, x_{3}\right) \in[0,1] \times[0, \widetilde{M}]^{3}, i=1,2,3$.

(C5) There exist $\widetilde{a}_{j i}, \widetilde{b}_{j i} \geq 0$ and $\widetilde{l}_{j}>0(i, j=1,2,3)$ such that

$$
\left(\begin{array}{l}
F_{1}\left(t, x_{1}, x_{2}, x_{3}\right) \\
F_{2}\left(t, x_{1}, x_{2}, x_{3}\right) \\
F_{3}\left(t, x_{1}, x_{2}, x_{3}\right)
\end{array}\right) \leq\left(\begin{array}{l}
\widetilde{a}_{11} x_{1}+\widetilde{a}_{12} x_{2}+\widetilde{a}_{13} x_{3}+\widetilde{l}_{1} \\
\widetilde{a}_{21} x_{1}+\widetilde{a}_{22} x_{2}+\widetilde{a}_{23} x_{3}+\widetilde{l}_{2} \\
\widetilde{a}_{31} x_{1}+\widetilde{a}_{32} x_{2}+\widetilde{a}_{33} x_{3}+\widetilde{l}_{3}
\end{array}\right), \forall\left(t, x_{1}, x_{2}, x_{3}\right) \in[0,1] \times \mathbb{R}^{+} \times \mathbb{R}^{+} \times \mathbb{R}^{+},
$$

and the matrix $\mathcal{M}_{2}$ is a $\mathbb{R}_{+}^{3}$-monotone matrix, where

$$
\mathcal{M}_{2}=\left(\begin{array}{ccc}
1-\kappa_{2} \widetilde{a}_{11} & -\kappa_{2} \widetilde{a}_{12} & -\kappa_{2} \widetilde{a}_{13} \\
-\kappa_{2} \widetilde{a}_{21} & 1-\kappa_{2} \widetilde{a}_{22} & -\kappa_{2} \widetilde{a}_{23} \\
-\kappa_{2} \widetilde{a}_{31} & -\kappa_{2} \widetilde{a}_{32} & 1-\kappa_{2} \widetilde{a}_{33}
\end{array}\right)
$$

(C6) There exists $\widetilde{Q}_{i}(t)$ in $[0,1]$, and $t_{0} \in(0,1)$ such that

$$
\int_{0}^{1} \varphi(t) \widetilde{Q}_{i}(t) d t>\widetilde{M} t_{0}^{1-\alpha}, \text { and } F_{i}\left(t, x_{1}, x_{2}, x_{3}\right) \geq \widetilde{Q}_{i}(t), \forall\left(t, x_{1}, x_{2}, x_{3}\right) \in[1, e] \times[0, \widetilde{M}]^{3}, i=1,2,3 .
$$

Let $B_{\rho}=\{u \in P:\|u\|<\rho\}$ for $\rho>0$ in the sequel. Then we easily have $\partial B_{\rho}=\{u \in P:\|u\|=$ $\rho\}, \bar{B}_{\rho}=\{u \in P:\|u\| \leq \rho\}$.

Theorem 1. Suppose that (C)-(C4) hold. Then (1) has a positive solution.

Proof. We first show that:

$$
(u, v, w) \neq T(u, v, w)+\lambda\left(\phi_{1}, \phi_{2}, \phi_{3}\right), \text { for } u, v, w \in \partial B_{R_{1}} \cap P, \lambda \geq 0,
$$

where $\phi_{i}(i=1,2,3)$ are given elements in cone $P_{0}$, and $R_{1}>\tilde{M}$. Argument by contrary, there exists $u, v, w \in \partial B_{R_{1}} \cap P$ and $\lambda_{0} \geq 0$ such that

$$
(u, v, w)=T(u, v, w)+\lambda_{0}\left(\phi_{1}, \phi_{2}, \phi_{3}\right), \text { for } u, v, w \in \partial B_{R_{1}} \cap P, \lambda \geq 0 .
$$


This implies that

$$
\left(\begin{array}{c}
u(t) \\
v(t) \\
w(t)
\end{array}\right)=\left(\begin{array}{l}
T_{1}(u, v, w)(t)+\lambda_{0} \phi_{1}(t) \\
T_{2}(u, v, w)(t)+\lambda_{0} \phi_{2}(t) \\
T_{3}(u, v, w)(t)+\lambda_{0} \phi_{3}(t)
\end{array}\right) \geq\left(\begin{array}{l}
\int_{0}^{1} G(t, s) F_{1}(s, u(s)-z(s), v(s)-z(s), w(s)-z(s)) d s \\
\int_{0}^{1} G(t, s) F_{2}(s, u(s)-z(s), v(s)-z(s), w(s)-z(s)) d s \\
\int_{0}^{1} G(t, s) F_{3}(s, u(s)-z(s), v(s)-z(s), w(s)-z(s)) d s
\end{array}\right) .
$$

Note that Lemma 7 we have

$$
u, v, w \in P_{0}
$$

From (C3) we have

$$
\left(\begin{array}{c}
u(t) \\
v(t) \\
w(t)
\end{array}\right) \geq\left(\begin{array}{l}
\int_{0}^{1} G(t, s)\left(a_{11}(u(s)-z(s))+a_{12}(v(s)-z(s))+a_{13}(w(s)-z(s))-l_{1}\right) d s \\
\int_{0}^{1} G(t, s)\left(a_{21}(u(s)-z(s))+a_{22}(v(s)-z(s))+a_{23}(w(s)-z(s))-l_{2}\right) d s \\
\int_{0}^{1} G(t, s)\left(a_{31}(u(s)-z(s))+a_{32}(v(s)-z(s))+a_{33}(w(s)-z(s))-l_{3}\right) d s
\end{array}\right) .
$$

Multiplying by $\varphi(t)$ for the above both sides, and integrating on $[0,1]$, by Lemma 4 we get

$$
\left(\begin{array}{l}
\int_{0}^{1} u(t) \varphi(t) d t \\
\int_{0}^{1} v(t) \varphi(t) d t \\
\int_{0}^{1} w(t) \varphi(t) d t
\end{array}\right) \geq\left(\begin{array}{l}
\int_{0}^{1} \kappa_{1} \varphi(t)\left(a_{11}(u(t)-z(t))+a_{12}(v(t)-z(t))+a_{13}(w(t)-z(t))\right) d t-l_{1} \kappa_{2}^{2} \\
\int_{0}^{1} \kappa_{1} \varphi(t)\left(a_{21}(u(t)-z(t))+a_{22}(v(t)-z(t))+a_{23}(w(t)-z(t))\right) d t-l_{2} \kappa_{2}^{2} \\
\int_{0}^{1} \kappa_{1} \varphi(t)\left(a_{31}(u(t)-z(t))+a_{32}(v(t)-z(t))+a_{33}(w(t)-z(t))\right) d t-l_{3} \kappa_{2}^{2}
\end{array}\right) .
$$

Consequently, we find

$$
\begin{aligned}
\left(\begin{array}{ccc}
\kappa_{1} a_{11}-1 & \kappa_{1} a_{12} & \kappa_{1} a_{13} \\
\kappa_{1} a_{21} & \kappa_{1} a_{22}-1 & \kappa_{1} a_{23} \\
\kappa_{1} a_{31} & \kappa_{1} a_{32} & \kappa_{1} a_{33}-1
\end{array}\right)\left(\begin{array}{c}
\int_{0}^{1} u(t) \varphi(t) d t \\
\int_{0}^{1} v(t) \varphi(t) d t \\
\int_{0}^{1} w(t) \varphi(t) d t
\end{array}\right) & \leq\left(\begin{array}{l}
\kappa_{1}\left(a_{11}+a_{12}+a_{13}\right) \int_{0}^{1} \varphi(t) z(t) d t+l_{1} \kappa_{2}^{2} \\
\kappa_{1}\left(a_{21}+a_{22}+a_{23}\right) \int_{0}^{1} \varphi(t) z(t) d t+l_{2} \kappa_{2}^{2} \\
\kappa_{1}\left(a_{31}+a_{32}+a_{33}\right) \int_{0}^{1} \varphi(t) z(t) d t+l_{3} \kappa_{2}^{2}
\end{array}\right) \\
& \leq\left(\begin{array}{c}
\kappa_{1}\left(a_{11}+a_{12}+a_{13}\right) M \kappa_{2}^{2}+l_{1} \kappa_{2}^{2} \\
\kappa_{1}\left(a_{21}+a_{22}+a_{23}\right) M \kappa_{2}^{2}+l_{2} \kappa_{2}^{2} \\
\kappa_{1}\left(a_{31}+a_{32}+a_{33}\right) M \kappa_{2}^{2}+l_{3} \kappa_{2}^{2}
\end{array}\right) .
\end{aligned}
$$


Therefore, we obtain

$$
\begin{aligned}
& \left(\begin{array}{l}
\int_{0}^{1} u(t) \varphi(t) d t \\
\int_{0}^{1} v(t) \varphi(t) d t \\
\int_{0}^{1} w(t) \varphi(t) d t
\end{array}\right) \leq\left(\begin{array}{ccc}
\kappa_{1} a_{11}-1 & \kappa_{1} a_{12} & \kappa_{1} a_{13} \\
\kappa_{1} a_{21} & \kappa_{1} a_{22}-1 & \kappa_{1} a_{23} \\
\kappa_{1} a_{31} & \kappa_{1} a_{32} & \kappa_{1} a_{33}-1
\end{array}\right)^{-1}\left(\begin{array}{c}
\kappa_{1}\left(a_{11}+a_{12}+a_{13}\right) M \kappa_{2}^{2}+l_{1} \kappa_{2}^{2} \\
\kappa_{1}\left(a_{21}+a_{22}+a_{23}\right) M \kappa_{2}^{2}+l_{2} \kappa_{2}^{2} \\
\kappa_{1}\left(a_{31}+a_{32}+a_{33}\right) M \kappa_{2}^{2}+l_{3} \kappa_{2}^{2}
\end{array}\right) \\
& =\frac{1}{\Delta_{1}}\left(\begin{array}{ccc}
\left(\kappa_{1} a_{22}-1\right)\left(\kappa_{1} a_{33}-1\right)-\kappa_{1}^{2} a_{23} a_{32} & \kappa_{1}^{2} a_{13} a_{32}-\kappa_{1} a_{12}\left(\kappa_{1} a_{33}-1\right) & \kappa_{1}^{2} a_{12} a_{23}-\kappa_{1} a_{13}\left(\kappa_{1} a_{22}-1\right) \\
\kappa_{1}^{2} a_{23} a_{31}-\kappa_{1} a_{21}\left(\kappa_{1} a_{33}-1\right) & \left(\kappa_{1} a_{11}-1\right)\left(\kappa_{1} a_{33}-1\right)-\kappa_{1}^{2} a_{13} a_{31} & \kappa_{1}^{2} a_{13} a_{21}-\kappa_{1} a_{23}\left(\kappa_{1} a_{11}-1\right) \\
\kappa_{1}^{2} a_{21} a_{32}-\kappa_{1} a_{31}\left(\kappa_{1} a_{22}-1\right) & \kappa_{1}^{2} a_{12} a_{31}-\kappa_{1} a_{32}\left(\kappa_{1} a_{11}-1\right) & \left(\kappa_{1} a_{11}-1\right)\left(\kappa_{1} a_{22}-1\right)-\kappa_{1}^{2} a_{12} a_{21}
\end{array}\right) \\
& \cdot\left(\begin{array}{c}
\kappa_{1}\left(a_{11}+a_{12}+a_{13}\right) M \kappa_{2}^{2}+l_{1} \kappa_{2}^{2} \\
\kappa_{1}\left(a_{21}+a_{22}+a_{23}\right) M \kappa_{2}^{2}+l_{2} \kappa_{2}^{2} \\
\kappa_{1}\left(a_{31}+a_{32}+a_{33}\right) M \kappa_{2}^{2}+l_{3} \kappa_{2}^{2}
\end{array}\right),
\end{aligned}
$$

where

$$
\Delta_{1}=\operatorname{det}\left(\begin{array}{ccc}
\kappa_{1} a_{11}-1 & \kappa_{1} a_{12} & \kappa_{1} a_{13} \\
\kappa_{1} a_{21} & \kappa_{1} a_{22}-1 & \kappa_{1} a_{23} \\
\kappa_{1} a_{31} & \kappa_{1} a_{32} & \kappa_{1} a_{33}-1
\end{array}\right) \text {. }
$$

As a result of this, there exist $\mathcal{N}_{i}>0(i=1,2,3)$ such that

$$
\left(\begin{array}{l}
\int_{0}^{1} u(t) \varphi(t) d t \\
\int_{0}^{1} v(t) \varphi(t) d t \\
\int_{0}^{1} w(t) \varphi(t) d t
\end{array}\right) \leq\left(\begin{array}{l}
\mathcal{N}_{1} \\
\mathcal{N}_{2} \\
\mathcal{N}_{3}
\end{array}\right)
$$

where $\mathcal{N}_{1}=\frac{1}{\Delta_{1}}\left[\left(\left(\kappa_{1} a_{22}-1\right)\left(\kappa_{1} a_{33}-1\right)-\kappa_{1}^{2} a_{23} a_{32}\right)\left(\kappa_{1}\left(a_{11}+a_{12}+a_{13}\right) M \kappa_{2}^{2}+l_{1} \kappa_{2}^{2}\right)+\left(\kappa_{1}^{2} a_{13} a_{32}-\right.\right.$ $\left.\kappa_{1} a_{12}\left(\kappa_{1} a_{33}-1\right)\right)\left(\kappa_{1}\left(a_{21}+a_{22}+a_{23}\right) M \kappa_{2}^{2}+l_{2} \kappa_{2}^{2}\right)+\left(\kappa_{1}^{2} a_{12} a_{23}-\kappa_{1} a_{13}\left(\kappa_{1} a_{22}-1\right)\right)\left(\kappa_{1}\left(a_{31}+a_{32}+\right.\right.$ $\left.\left.\left.a_{33}\right) M \kappa_{2}^{2}+l_{3} \kappa_{2}^{2}\right)\right], \mathcal{N}_{2}=\frac{1}{\Delta_{1}}\left[\left(\kappa_{1}^{2} a_{23} a_{31}-\kappa_{1} a_{21}\left(\kappa_{1} a_{33}-1\right)\right)\left(\kappa_{1}\left(a_{11}+a_{12}+a_{13}\right) M \kappa_{2}^{2}+l_{1} \kappa_{2}^{2}\right)+\left(\left(\kappa_{1} a_{11}-\right.\right.\right.$ 1) $\left.\left(\kappa_{1} a_{33}-1\right)-\kappa_{1}^{2} a_{13} a_{31}\right)\left(\kappa_{1}\left(a_{21}+a_{22}+a_{23}\right) M \kappa_{2}^{2}+l_{2} \kappa_{2}^{2}\right)+\left(\kappa_{1}^{2} a_{13} a_{21}-\kappa_{1} a_{23}\left(\kappa_{1} a_{11}-1\right)\right)\left(\kappa_{1}\left(a_{31}+a_{32}+\right.\right.$ $\left.\left.\left.a_{33}\right) M \kappa_{2}^{2}+l_{3} \kappa_{2}^{2}\right)\right], \mathcal{N}_{3}=\frac{1}{\Delta_{1}}\left[\left(\kappa_{1}^{2} a_{21} a_{32}-\kappa_{1} a_{31}\left(\kappa_{1} a_{22}-1\right)\right)\left(\kappa_{1}\left(a_{11}+a_{12}+a_{13}\right) M \kappa_{2}^{2}+l_{1} \kappa_{2}^{2}\right)+\left(\kappa_{1}^{2} a_{12} a_{31}-\right.\right.$ $\left.\kappa_{1} a_{32}\left(\kappa_{1} a_{11}-1\right)\right)\left(\kappa_{1}\left(a_{21}+a_{22}+a_{23}\right) M \kappa_{2}^{2}+l_{2} \kappa_{2}^{2}\right)+\left(\left(\kappa_{1} a_{11}-1\right)\left(\kappa_{1} a_{22}-1\right)-\kappa_{1}^{2} a_{21} a_{21}\right)\left(\kappa_{1}\left(a_{31}+a_{32}+\right.\right.$ $\left.\left.\left.a_{33}\right) M \kappa_{2}^{2}+l_{3} \kappa_{2}^{2}\right)\right]$.

Note that (22), we have

$$
\left(\begin{array}{l}
\|u\| \\
\|v\| \\
\|w\|
\end{array}\right) \leq\left(\begin{array}{l}
\mathcal{N}_{1} \kappa_{1}^{-1} \\
\mathcal{N}_{2} \kappa_{1}^{-1} \\
\mathcal{N}_{3} \kappa_{1}^{-1}
\end{array}\right)
$$

Therefore, we can choose $R_{1}>\max \left\{\tilde{M}, \mathcal{N}_{1} \kappa_{1}^{-1}, \mathcal{N}_{2} \kappa_{1}^{-1}, \mathcal{N}_{3} \kappa_{1}^{-1}\right\}$ such that when $u, v, w \in \partial B_{R_{1}} \cap P,(21)$ is not satisfied. This also indicates that (20) holds for $u, v, w \in \partial B_{R_{1}} \cap P$, and Lemma 8 indicates that

$$
i\left(T, B_{R_{1}} \cap(P \times P \times P), P \times P \times P\right)=0 .
$$

On the other hand, we prove that

$$
(u, v, w) \neq \lambda T(u, v, w), \text { for } u, v, w \in \partial B_{\widetilde{M}} \cap P, \lambda \in[0,1] \text {. }
$$


If this claim is not true, there exist $u, v, w \in \partial B_{\widetilde{M}} \cap P, \lambda_{1} \in[0,1]$ such that

$$
(u, v, w)=\lambda_{1} T(u, v, w) .
$$

This implies that

$$
\|u\| \leq\left\|T_{1}(u, v, w)\right\|,\|v\| \leq\left\|T_{2}(u, v, w)\right\|, \text { and }\|w\| \leq\left\|T_{3}(u, v, w)\right\| .
$$

However, from (C4) we have

$$
\begin{aligned}
T_{1}(u, v, w)(t) & =\int_{0}^{1} G(t, s) F_{1}(s, u(s)-z(s), v(s)-z(s), w(s)-z(s)) d s \\
& \leq \int_{0}^{1} \varphi(s) Q_{1}(s) \frac{d s}{s} \\
& <\widetilde{M}
\end{aligned}
$$

Note that by (C4), $\|u\|=\tilde{M}$. Hence, we obtain $\left\|T_{1}(u, v, w)\right\|<\|u\|$. Similarly, $\left\|T_{2}(u, v, w)\right\|<\|v\|$ and $\left\|T_{3}(u, v, w)\right\|<\|w\|$. This has a contradiction. Hence (24) holds. By Lemma 9 we get

$$
i\left(T, B_{\widetilde{M}} \cap(P \times P \times P), P \times P \times P\right)=1 .
$$

By use of (23) and (25) we can calculate

$$
\begin{aligned}
& i\left(T,\left(B_{R_{1}} \backslash \bar{B}_{\widetilde{M}}\right) \cap(P \times P \times P), P \times P \times P\right) \\
& \quad=i\left(T, B_{R_{1}} \cap(P \times P \times P), P \times P \times P\right)-i\left(T, B_{\widetilde{M}} \cap(P \times P \times P), P \times P \times P\right) \\
& \quad=-1 .
\end{aligned}
$$

Therefore, $T$ has a fixed point $\left(u^{*}, v^{*}, w^{*}\right)$ on $\left(B_{R_{1}} \backslash \bar{B}_{\widetilde{M}}\right) \cap(P \times P \times P)$. Consequently, $\left(u^{*}-z, v^{*}-\right.$ $\left.z, w^{*}-z\right)$ is a positive solution for (1), i.e., (1) has a positive solution.

Theorem 2. Suppose that (C0)-(C2), (C5)-(C6) hold. Then (1) has a positive solution.

Proof. We first claim that:

$$
(u, v, w) \neq \lambda T(u, v, w), \text { for } u, v, w \in \partial B_{R_{2}} \cap P, \lambda \in[0,1],
$$

where $R_{2}>\tilde{M}$. If this claim does not hold, there exist $u, v, w \in \partial B_{R_{2}} \cap P, \lambda_{2} \in[0,1]$ such that

$$
(u, v, w)=\lambda_{2} T(u, v, w) .
$$

This indicates that

$$
\left(\begin{array}{c}
u(t) \\
v(t) \\
w(t)
\end{array}\right)=\left(\begin{array}{l}
\lambda_{2} T_{1}(u, v, w)(t) \\
\lambda_{2} T_{2}(u, v, w)(t) \\
\lambda_{2} T_{3}(u, v, w)(t)
\end{array}\right) \leq\left(\begin{array}{l}
\int_{0}^{1} G(t, s) F_{1}(s, u(s)-z(s), v(s)-z(s), w(s)-z(s)) d s \\
\int_{0}^{1} G(t, s) F_{2}(s, u(s)-z(s), v(s)-z(s), w(s)-z(s)) d s \\
\int_{0}^{1} G(t, s) F_{3}(s, u(s)-z(s), v(s)-z(s), w(s)-z(s)) d s
\end{array}\right) .
$$


Using Lemma 7, we know $u, v, w \in P_{0}$. By virtue of (C5), we obtain

$$
\left(\begin{array}{c}
u(t) \\
v(t) \\
w(t)
\end{array}\right) \leq\left(\begin{array}{c}
\int_{0}^{1} G(t, s)\left(\widetilde{a}_{11}(u(s)-z(s))+\widetilde{a}_{12}(v(s)-z(s))+\widetilde{a}_{13}(w(s)-z(s))+\widetilde{l}_{1}\right) d s \\
\int_{0}^{1} G(t, s)\left(\widetilde{a}_{21}(u(s)-z(s))+\widetilde{a}_{22}(v(s)-z(s))+\widetilde{a}_{23}(w(s)-z(s))+\widetilde{l}_{2}\right) d s \\
\int_{0}^{1} G(t, s)\left(\widetilde{a}_{31}(u(s)-z(s))+\widetilde{a}_{32}(v(s)-z(s))+\widetilde{a}_{33}(w(s)-z(s))+\widetilde{l}_{3}\right) d s
\end{array}\right) .
$$

Multiplying by $\varphi(t)$, and integrating over [0,1], Lemma 4 enables us to get

$$
\begin{aligned}
\left(\begin{array}{l}
\int_{0}^{1} u(t) \varphi(t) d t \\
\int_{0}^{1} v(t) \varphi(t) d t \\
\int_{0}^{1} w(t) \varphi(t) d t
\end{array}\right) \leq & \left(\begin{array}{l}
\int_{0}^{1} \kappa_{2} \varphi(t)\left(\widetilde{a}_{11}(u(t)-z(t))+\widetilde{a}_{12}(v(t)-z(t))+\widetilde{a}_{13}(w(t)-z(t))+\widetilde{l}_{1}\right) d t \\
\int_{0}^{1} \kappa_{2} \varphi(t)\left(\widetilde{a}_{21}(u(t)-z(t))+\widetilde{a}_{22}(v(t)-z(t))+\widetilde{a}_{23}(w(t)-z(t))+\widetilde{l}_{2}\right) d t \\
\int_{0}^{1} \kappa_{2} \varphi(t)\left(\widetilde{a}_{31}(u(t)-z(t))+\widetilde{a}_{32}(v(t)-z(t))+\widetilde{a}_{33}(w(t)-z(t))+\widetilde{l}_{3}\right) d t
\end{array}\right) \\
\leq & \left(\begin{array}{l}
\int_{0}^{1} \kappa_{2} \varphi(t)\left(\widetilde{a}_{11} u(t)+\widetilde{a}_{12} v(t)+\widetilde{a}_{13} w(t)\right) d t+\widetilde{l}_{1} \kappa_{2}^{2} \\
\int_{0}^{1} \kappa_{2} \varphi(t)\left(\widetilde{a}_{21} u(t)+\widetilde{a}_{22} v(t)+\widetilde{a}_{23} w(t)\right) d t+\widetilde{l}_{2} \kappa_{2}^{2} \\
\int_{0}^{1} \kappa_{2} \varphi(t)\left(\widetilde{a}_{31} u(t)+\widetilde{a}_{32} v(t)+\widetilde{a}_{33} w(t)\right) d t+\widetilde{l}_{3} \kappa_{2}^{2}
\end{array}\right) .
\end{aligned}
$$

Therefore, we find

$$
\left(\begin{array}{ccc}
1-\kappa_{2} \widetilde{a}_{11} & -\kappa_{2} \widetilde{a}_{12} & -\kappa_{2} \widetilde{a}_{13} \\
-\kappa_{2} \widetilde{a}_{21} & 1-\kappa_{2} \widetilde{a}_{22} & -\kappa_{2} \widetilde{a}_{23} \\
-\kappa_{2} \widetilde{a}_{31} & -\kappa_{2} \widetilde{a}_{32} & 1-\kappa_{2} \widetilde{a}_{33}
\end{array}\right)\left(\begin{array}{c}
\int_{0}^{1} u(t) \varphi(t) d t \\
\int_{0}^{1} v(t) \varphi(t) d t \\
\int_{0}^{1} w(t) \varphi(t) d t
\end{array}\right) \leq\left(\begin{array}{c}
\widetilde{l}_{1} \kappa_{2}^{2} \\
\widetilde{l}_{2} \kappa_{2}^{2} \\
\widetilde{l}_{3} \kappa_{2}^{2}
\end{array}\right)
$$

and

$$
\begin{aligned}
& \left(\begin{array}{l}
\int_{0}^{1} u(t) \varphi(t) d t \\
\int_{0}^{1} v(t) \varphi(t) d t \\
\int_{0}^{1} w(t) \varphi(t) d t
\end{array}\right) \leq\left(\begin{array}{ccc}
1-\kappa_{2} \widetilde{a}_{11} & -\kappa_{2} \widetilde{a}_{12} & -\kappa_{2} \widetilde{a}_{13} \\
-\kappa_{2} \widetilde{a}_{21} & 1-\kappa_{2} \widetilde{a}_{22} & -\kappa_{2} \widetilde{a}_{23} \\
-\kappa_{2} \widetilde{a}_{31} & -\kappa_{2} \widetilde{a}_{32} & 1-\kappa_{2} \widetilde{a}_{33}
\end{array}\right)^{-1}\left(\begin{array}{l}
\widetilde{l}_{1} \kappa_{2}^{2} \\
\widetilde{l}_{2} \kappa_{2}^{2} \\
\tilde{l}_{3} \kappa_{2}^{2}
\end{array}\right) \\
& =\frac{1}{\Delta_{2}}\left(\begin{array}{ccc}
\left(1-\kappa_{2} \widetilde{a}_{22}\right)\left(1-\kappa_{2} \widetilde{a}_{33}\right)-\kappa_{2}^{2} \widetilde{a}_{23} \widetilde{a}_{32} & \kappa_{2}^{2} \widetilde{a}_{13} \widetilde{a}_{32}+\kappa_{2} \widetilde{a}_{12}\left(1-\kappa_{2} \widetilde{a}_{33}\right) & \kappa_{2}^{2} \widetilde{a}_{12} \widetilde{a}_{23}+\kappa_{2} \widetilde{a}_{13}\left(1-\kappa_{2} \widetilde{a}_{22}\right) \\
\kappa_{2}^{2} \widetilde{a}_{23} \widetilde{a}_{31}+\kappa_{2} \widetilde{a}_{21}\left(1-\kappa_{2} \widetilde{a}_{33}\right) & \left(1-\kappa_{2} \widetilde{a}_{11}\right)\left(1-\kappa_{2} \widetilde{a}_{33}\right)-\kappa_{2}^{2} \widetilde{a}_{13} \widetilde{a}_{31} & \kappa_{2}^{2} \widetilde{a}_{13} \widetilde{a}_{21}+\kappa_{2} \widetilde{a}_{23}\left(1-\kappa_{2} \widetilde{a}_{11}\right) \\
\kappa_{2}^{2} \widetilde{a}_{21} \widetilde{a}_{32}+\kappa_{2} \widetilde{a}_{31}\left(1-\kappa_{2} \widetilde{a}_{22}\right) & \kappa_{2}^{2} \widetilde{a}_{12} \widetilde{a}_{31}+\kappa_{2} \widetilde{a}_{32}\left(1-\kappa_{2} \widetilde{a}_{11}\right) & \left(1-\kappa_{2} \widetilde{a}_{11}\right)\left(1-\kappa_{2} \widetilde{a}_{22}\right)-\kappa_{2}^{2} \widetilde{a}_{12} \widetilde{a}_{21}
\end{array}\right) \\
& \qquad\left(\begin{array}{c}
\widetilde{l}_{1} \kappa_{2}^{2} \\
\widetilde{l}_{2} \kappa_{2}^{2} \\
\widetilde{l}_{3} \kappa_{2}^{2}
\end{array}\right),
\end{aligned}
$$

where

$$
\Delta_{2}=\operatorname{det}\left(\begin{array}{ccc}
1-\kappa_{2} \widetilde{a}_{11} & -\kappa_{2} \widetilde{a}_{12} & -\kappa_{2} \widetilde{a}_{13} \\
-\kappa_{2} \widetilde{a}_{21} & 1-\kappa_{2} \widetilde{a}_{22} & -\kappa_{2} \widetilde{a}_{23} \\
-\kappa_{2} \widetilde{a}_{31} & -\kappa_{2} \widetilde{a}_{32} & 1-\kappa_{2} \widetilde{a}_{33}
\end{array}\right) \text {. }
$$


Hence, there exist $\mathcal{N}_{i}>0(i=4,5,6)$ such that

$$
\left(\begin{array}{l}
\int_{0}^{1} u(t) \varphi(t) d t \\
\int_{0}^{1} v(t) \varphi(t) d t \\
\int_{0}^{1} w(t) \varphi(t) d t
\end{array}\right) \leq\left(\begin{array}{l}
\mathcal{N}_{4} \\
\mathcal{N}_{5} \\
\mathcal{N}_{6}
\end{array}\right)
$$

where $\mathcal{N}_{4}=\frac{\kappa_{2}^{2}}{\Delta_{2}}\left[\widetilde{l}_{1}\left(\left(1-\kappa_{2} \widetilde{a}_{22}\right)\left(1-\kappa_{2} \widetilde{a}_{33}\right)-\kappa_{2}^{2} \widetilde{a}_{23} \widetilde{a}_{32}\right)+\widetilde{l}_{2}\left(\kappa_{2}^{2} \widetilde{a}_{13} \widetilde{a}_{32}+\kappa_{2} \widetilde{a}_{12}\left(1-\kappa_{2} \widetilde{a}_{33}\right)\right)+\widetilde{l}_{3}\left(\kappa_{2}^{2} \widetilde{a}_{12} \widetilde{a}_{23}+\right.\right.$ $\left.\left.\kappa_{2} \widetilde{a}_{13}\left(1-\kappa_{2} \widetilde{a}_{22}\right)\right)\right], \mathcal{N}_{5}=\frac{\kappa_{2}^{2}}{\Delta_{2}}\left[\widetilde{l}_{1}\left(\kappa_{2}^{2} \widetilde{a}_{23} \widetilde{a}_{31}+\kappa_{2} \widetilde{a}_{21}\left(1-\kappa_{2} \widetilde{a}_{33}\right)\right)+\widetilde{l}_{2}\left(\left(1-\kappa_{2} \widetilde{a}_{11}\right)\left(1-\kappa_{2} \widetilde{a}_{33}\right)-\kappa_{2}^{2} \widetilde{a}_{13} \widetilde{a}_{31}\right)+\right.$ $\left.\widetilde{l}_{3}\left(\kappa_{2}^{2} \widetilde{a}_{13} \widetilde{a}_{21}+\kappa_{2} \widetilde{a}_{23}\left(1-\kappa_{2} \widetilde{a}_{11}\right)\right)\right], \mathcal{N}_{6}=\frac{\kappa_{2}^{2}}{\Delta_{2}}\left[\widetilde{l}_{1}\left(\kappa_{2}^{2} \widetilde{a}_{21} \widetilde{a}_{32}+\kappa_{2} \widetilde{a}_{31}\left(1-\kappa_{2} \widetilde{a}_{22}\right)\right)+\widetilde{l}_{2}\left(\kappa_{2}^{2} \widetilde{a}_{12} \widetilde{a}_{31}+\kappa_{2} \widetilde{a}_{32}(1-\right.\right.$ $\left.\left.\left.\kappa_{2} \widetilde{a}_{11}\right)\right)+\widetilde{l}_{3}\left(\left(1-\kappa_{2} \widetilde{a}_{11}\right)\left(1-\kappa_{2} \widetilde{a}_{22}\right)-\kappa_{2}^{2} \widetilde{a}_{12} \widetilde{a}_{21}\right)\right]$. Note that $u, v, w \in P_{0}$, we have

$$
\left(\begin{array}{l}
\|u\| \\
\|v\| \\
\|w\|
\end{array}\right) \leq\left(\begin{array}{l}
\mathcal{N}_{4} \kappa_{1}^{-1} \\
\mathcal{N}_{5} \kappa_{1}^{-1} \\
\mathcal{N}_{6} \kappa_{1}^{-1}
\end{array}\right) .
$$

Therefore, we can choose $R_{2}>\max \left\{\tilde{M}, \mathcal{N}_{4} \kappa_{1}^{-1}, \mathcal{N}_{5} \kappa_{1}^{-1}, \mathcal{N}_{6} \kappa_{1}^{-1}\right\}$ such that when $u, v, w \in \partial B_{R_{2}} \cap P$, (27) is not satisfied. This also indicates that (26) holds for $u, v, w \in \partial B_{R_{2}} \cap P$, and by Lemma 9 we get

$$
i\left(T, B_{R_{2}} \cap(P \times P \times P), P \times P \times P\right)=1 .
$$

On the other hand, we prove that

$$
(u, v, w) \neq T(u, v, w)+\lambda\left(\widetilde{\phi}_{1}, \widetilde{\phi}_{2}, \widetilde{\phi}_{3}\right), \text { for } u, v, w \in \partial B_{\widetilde{M}} \cap P, \forall \lambda \geq 0,
$$

where $\widetilde{\phi}_{i} \in P(i=1,2,3)$ are fixed elements. Otherwise, there exist $u, v, w \in \partial B_{\widetilde{M}} \cap P, \lambda_{3} \geq 0$ such that

$$
(u, v, w)=T(u, v, w)+\lambda_{3}\left(\widetilde{\phi}_{1}, \widetilde{\phi}_{2}, \widetilde{\phi}_{3}\right) .
$$

This implies that

$$
\left(\begin{array}{c}
\|u\| \\
\|v\| \\
\|w\|
\end{array}\right) \geq\left(\begin{array}{l}
\left\|T_{1}(u, v, w)\right\| \\
\left\|T_{2}(u, v, w)\right\| \\
\left\|T_{3}(u, v, w)\right\|
\end{array}\right) .
$$

However, from (C6) we have

$$
\begin{aligned}
T_{i}(u, v, w)\left(t_{0}\right) & =\int_{0}^{1} G\left(t_{0}, s\right) F_{i}(s, u(s)-z(s), v(s)-z(s), w(s)-z(s)) d s \\
& \geq t_{0}^{\alpha-1} \int_{0}^{1} \varphi(s) \widetilde{Q}_{i}(s) d s \\
& >\widetilde{M}, i=1,2,3 .
\end{aligned}
$$

Note that from (C6), we have $\|u\|=\widetilde{M}$. Hence, we obtain

$$
\left(\begin{array}{l}
\left\|T_{1}(u, v, w)\right\| \\
\left\|T_{2}(u, v, w)\right\| \\
\left\|T_{3}(u, v, w)\right\|
\end{array}\right) \geq\left(\begin{array}{l}
T_{1}(u, v, w)\left(t_{0}\right) \\
T_{2}(u, v, w)\left(t_{0}\right) \\
T_{3}(u, v, w)\left(t_{0}\right)
\end{array}\right)>\left(\begin{array}{l}
\|u\| \\
\|v\| \\
\|w\|
\end{array}\right) .
$$


This has a contradiction with (30), and thus (29) holds. By Lemma 8 we find

$$
i\left(T, B_{\widetilde{M}} \cap(P \times P \times P), P \times P \times P\right)=0 .
$$

From (28) and (31) we can calculate

$$
\begin{aligned}
& i\left(T,\left(B_{R_{2}} \backslash \bar{B}_{\tilde{M}}\right) \cap(P \times P \times P), P \times P \times P\right) \\
& \quad=i\left(T, B_{R_{2}} \cap(P \times P \times P), P \times P \times P\right)-i\left(T, B_{\widetilde{M}} \cap(P \times P \times P), P \times P \times P\right) \\
& \quad=1 .
\end{aligned}
$$

Therefore $T$ has a fixed point $\left(u^{*}, v^{*}, w^{*}\right)$ on $\left(B_{R_{2}} \backslash \bar{B}_{\widetilde{M}}\right) \cap(P \times P \times P)$. Therefore, $\left(u^{*}-z, v^{*}-z, w^{*}-z\right)$ is a positive solution for (1), i.e., (1) has a positive solution.

Let $n=4, \alpha=3.5, p=1.5, q=0.5$, and $h(t)=t, t \in[0,1]$. Then we have $A=2.91$, and $\int_{0}^{1} h(t) g_{2}(t, s) d t=\frac{5}{24} s-\frac{1}{4} s^{2}+\frac{1}{24} s^{4}, s \in[0,1]$. This implies that (C0)-(C1) hold. Moreover, we can calculate

$$
\kappa_{1}=0.017, \kappa_{2}=0.075, \widetilde{M}=0.16 M \text {. }
$$

Example 1. Let $\kappa_{1} a_{11}-1=\kappa_{1} a_{22}-1=\kappa_{1} a_{33}-1=\kappa_{1}$, and we have $a_{11}=a_{22}=a_{33}=\frac{\kappa_{1}+1}{\kappa_{1}}=59.82$. Moreover, we take the matrix

$$
\left(\begin{array}{lll}
a_{11} & a_{12} & a_{13} \\
a_{21} & a_{22} & a_{23} \\
a_{31} & a_{32} & a_{33}
\end{array}\right)=\left(\begin{array}{ccc}
59.82 & 0 & 0 \\
0 & 59.82 & 0 \\
0 & 0 & 59.82
\end{array}\right)
$$

and

$$
\left(\begin{array}{l}
F_{1}\left(t, x_{1}, x_{2}, x_{3}\right) \\
F_{2}\left(t, x_{1}, x_{2}, x_{3}\right) \\
F_{3}\left(t, x_{1}, x_{2}, x_{3}\right)
\end{array}\right)=\left(\begin{array}{c}
2 M(9.57 M)^{-\gamma_{1}}\left(a_{11} x_{1}+a_{12} x_{2}+a_{13} x_{3}\right)^{\gamma_{1}} \\
1.8 M(9.57 M)^{-\gamma_{2}}\left(a_{21} x_{1}+a_{22} x_{2}+a_{23} x_{3}\right)^{\gamma_{2}} \\
1.5 M(9.57 M)^{-\gamma_{3}}\left(a_{31} x_{1}+a_{32} x_{2}+a_{33} x_{3}\right)^{\gamma_{3}}
\end{array}\right), \forall\left(t, x_{1}, x_{2}, x_{3}\right) \in[0,1] \times \mathbb{R}^{+} \times \mathbb{R}^{+} \times \mathbb{R}^{+},
$$

where $\gamma_{i}>1(i=1,2,3)$. Note that

$$
\mathcal{M}_{1}=\left(\begin{array}{ccc}
\kappa_{1} a_{11}-1 & \kappa_{1} a_{12} & \kappa_{1} a_{13} \\
\kappa_{1} a_{21} & \kappa_{1} a_{22}-1 & \kappa_{1} a_{23} \\
\kappa_{1} a_{31} & \kappa_{1} a_{32} & \kappa_{1} a_{33}-1
\end{array}\right)=\left(\begin{array}{ccc}
\kappa_{1} & 0 & 0 \\
0 & \kappa_{1} & 0 \\
0 & 0 & \kappa_{1}
\end{array}\right) .
$$

Hence, $\mathcal{M}_{1}$ is a $\mathbb{R}_{+}^{3}$-monotone matrix. Furthermore, for all $t \in[0,1]$ we have

$$
\begin{aligned}
& \liminf _{a_{11} x_{1}+a_{12} x_{2}+a_{13} x_{3} \rightarrow+\infty} \frac{F_{1}\left(x_{1}, x_{2}, x_{3}\right)}{a_{11} x_{1}+a_{12} x_{2}+a_{13} x_{3}}=\liminf _{a_{11} x_{1}+a_{12} x_{2}+a_{13} x_{3} \rightarrow+\infty} \frac{2 M(9.57 M)^{-\gamma_{1}}\left(a_{11} x_{1}+a_{12} x_{2}+a_{13} x_{3}\right)^{\gamma_{1}}}{a_{11} x_{1}+a_{12} x_{2}+a_{13} x_{3}}=+\infty, \\
& \liminf _{a_{21} x_{1}+a_{22} x_{2}+a_{23} x_{3} \rightarrow+\infty} \frac{F_{2}\left(x_{1}, x_{2}, x_{3}\right)}{a_{21} x_{1}+a_{22} x_{2}+a_{23} x_{3}}=\liminf _{a_{21} x_{1}+a_{22} x_{2}+a_{23} x_{3} \rightarrow+\infty} \frac{1.8 M(9.57 M)^{-\gamma_{2}}\left(a_{21} x_{1}+a_{22} x_{2}+a_{23} x_{3}\right)^{\gamma_{2}}}{a_{21} x_{1}+a_{22} x_{2}+a_{23} x_{3}}=+\infty \text {, } \\
& \liminf _{a_{31} x_{1}+a_{32} x_{2}+a_{33} x_{3} \rightarrow+\infty} \frac{F_{3}\left(x_{1}, x_{2}, x_{3}\right)}{a_{31} x_{1}+a_{32} x_{2}+a_{33} x_{3}}=\liminf _{a_{31} x_{1}+a_{32} x_{2}+a_{33} x_{3} \rightarrow+\infty} \frac{1.5 M(9.57 M)^{-\gamma_{3}}\left(a_{31} x_{1}+a_{32} x_{2}+a_{33} x_{3}\right)^{\gamma_{3}}}{a_{31} x_{1}+a_{32} x_{2}+a_{33} x_{3}}=+\infty .
\end{aligned}
$$

On the other hand, if $\left(t, x_{1}, x_{2}, x_{3}\right) \in[0,1] \times[0,0.16 M]^{3}$, we have

$$
F_{1} \leq 2 M, F_{2} \leq 1.8 M, F_{3} \leq 1.5 M \text {. }
$$


If we choose $Q_{1}(t) \equiv 2 M, Q_{2}(t) \equiv 1.8 M, Q_{3}(t) \equiv 1.5 M$ for $t \in[0,1]$, and we have

$$
\int_{0}^{1} \varphi(t) Q_{i}(t) d t \leq \int_{0}^{1} \varphi(t) Q_{1}(t) d t=2 \kappa_{2} M=0.15 M<\widetilde{M}, i=1,2,3 .
$$

Therefore, (C3)-(C4) hold.

Example 2. Let $t_{0}=0.5, \widetilde{Q}_{1}(t)=13 M, \widetilde{Q}_{2}(t)=14 M, \widetilde{Q}_{3}(t)=15 M$ for $t \in[0,1]$, and

$$
\left(\begin{array}{lll}
\widetilde{a}_{11} & \widetilde{a}_{12} & \widetilde{a}_{13} \\
\widetilde{a}_{21} & \widetilde{a}_{22} & \widetilde{a}_{23} \\
\widetilde{a}_{31} & \widetilde{a}_{32} & \widetilde{a}_{33}
\end{array}\right)=\left(\begin{array}{lll}
2 & 5 & 3 \\
8 & 3 & 4 \\
6 & 3 & 4
\end{array}\right)
$$

and

$$
\left(\begin{array}{l}
F_{1}\left(t, x_{1}, x_{2}, x_{3}\right) \\
F_{2}\left(t, x_{1}, x_{2}, x_{3}\right) \\
F_{3}\left(t, x_{1}, x_{2}, x_{3}\right)
\end{array}\right)=\left(\begin{array}{c}
13 M e^{1.6 M} e^{-2 x_{1}-5 x_{2}-3 x_{3}} \\
14 M e^{2.4 M} e^{-8 x_{1}-3 x_{2}-4 x_{3}} \\
15 M e^{2.08 M} e^{-6 x_{1}-3 x_{2}-4 x_{3}}
\end{array}\right), \forall\left(t, x_{1}, x_{2}, x_{3}\right) \in[0,1] \times \mathbb{R}^{+} \times \mathbb{R}^{+} \times \mathbb{R}^{+}
$$

Then if $\left(t, x_{1}, x_{2}, x_{3}\right) \in[0,1] \times[0,0.16 M]^{3}$, we have $F_{1} \geq 13 M, F_{2} \geq 14 M, F_{3} \geq 15 M$, and $\int_{0}^{1} \varphi(t) \widetilde{Q}_{i}(t) d t \geq \int_{0}^{1} \varphi(t) \widetilde{Q}_{1}(t) d t=13 \kappa_{2} M>0.16 \times 5.6569 M$.

On the other hand, we can calculate

$$
\operatorname{det} \mathcal{M}_{2}=\operatorname{det}\left(\begin{array}{ccc}
1-0.075 \times 2 & -0.075 \times 5 & -0.075 \times 3 \\
-0.075 \times 8 & 1-0.075 \times 3 & -0.075 \times 4 \\
-0.075 \times 6 & -0.075 \times 3 & 1-0.075 \times 4
\end{array}\right)=0.0868
$$

and

$$
\mathcal{M}_{2}^{-1}=\frac{1}{0.0868}\left(\begin{array}{ccc}
0.475 & 0.313 & 0.287 \\
0.555 & 0.494 & 0.39 \\
0.484 & 0.36 & 0.434
\end{array}\right)
$$

Consequently, $\mathcal{M}_{2}$ is a $\mathbb{R}_{+}^{3}$-monotone matrix. Furthermore, for all $t \in[0,1]$ we have

$$
\begin{aligned}
& \limsup _{\widetilde{a}_{11} x_{1}+\widetilde{a}_{12} x_{2}+\widetilde{a}_{13} x_{3} \rightarrow+\infty} \frac{F_{1}\left(t, x_{1}, x_{2}, x_{3}\right)}{\widetilde{a}_{11} x_{1}+\widetilde{a}_{12} x_{2}+\widetilde{a}_{13} x_{3}}=\underset{\widetilde{a}_{11} x_{1}+\widetilde{a}_{12} x_{2}+\widetilde{a}_{13} x_{3} \rightarrow+\infty}{\limsup } \frac{13 M e^{1.6 M} e^{-\widetilde{a}_{11} x_{1}-\widetilde{a}_{12} x_{2}-\widetilde{a}_{13} x_{3}}}{\widetilde{a}_{11} x_{1}+\widetilde{a}_{12} x_{2}+\widetilde{a}_{13} x_{3}}=0, \\
& \limsup _{\widetilde{a}_{21} x_{1}+\widetilde{a}_{22} x_{2}+\widetilde{a}_{23} x_{3} \rightarrow+\infty} \frac{F_{2}\left(t, x_{1}, x_{2}, x_{3}\right)}{\widetilde{a}_{21} x_{1}+\widetilde{a}_{22} x_{2}+\widetilde{a}_{23} x_{3}}=\underset{\widetilde{a}_{21} x_{1}+\widetilde{a}_{22} x_{2}+\widetilde{a}_{23} x_{3} \rightarrow+\infty}{\limsup } \frac{14 M e^{2.4 M} e^{-\widetilde{a}_{21} x_{1}-\widetilde{a}_{22} x_{2}-\widetilde{a}_{23} x_{3}}}{\widetilde{a}_{21} x_{1}+\widetilde{a}_{22} x_{2}+\widetilde{a}_{23} x_{3}}=0, \\
& \limsup _{\widetilde{a}_{31} x_{1}+\widetilde{a}_{32} x_{2}+\widetilde{a}_{33} x_{3} \rightarrow+\infty} \frac{F_{3}\left(t, x_{1}, x_{2}, x_{3}\right)}{\widetilde{a}_{31} x_{1}+\widetilde{a}_{32} x_{2}+\widetilde{a}_{33} x_{3}}=\underset{\widetilde{a}_{31} x_{1}+\widetilde{a}_{32} x_{2}+\widetilde{a}_{33} x_{3} \rightarrow+\infty}{\limsup } \frac{15 M e^{2.08 M} e^{-\widetilde{a}_{31} x_{1}-\widetilde{a}_{32} x_{2}-\widetilde{a}_{33} x_{3}}}{\widetilde{a}_{31} x_{1}+\widetilde{a}_{32} x_{2}+\widetilde{a}_{33} x_{3}}=0 .
\end{aligned}
$$

As a result, (C5)-(C6) hold.

\section{Conclusions}

In this paper, we utilize the index of fixed point to research the positive solutions for the system of Riemann-Liouville type fractional boundary value problems (1). We first investigate corresponding operator equations for (1), and then establish some coupling behaviors for our nonlinearities $f_{i}(i=1,2,3)$ by virtue of nonnegative matrix theory, which ensure that our nonlinearities can grow superlinearly and sublinearly at $\infty$. 
Author Contributions: Conceptualization, Y.D. and J.X.; methodology, J.X.; software, Y.D.; validation, Y.D., J.X. and Z.F.; formal analysis, J.X.; investigation, Y.D.; resources, J.X.; data curation, J.X.; writing-original draft preparation, Y.D.; writing-review and editing, J.X.; visualization, J.X.; supervision, J.X. and Z.F.; project administration, J.X.; funding acquisition, J.X.

Funding: This work is supported by the National Natural Science Foundation of China (Grant No. 11601048), and the Natural Science Foundation of Chongqing Normal University (Grant No. 16XYY24).

Conflicts of Interest: The authors declare no conflict of interest.

\section{References}

1. Kilbas, A.A.; Srivastava, H.M.; Trujillo, J.J. Theory and Applications of Fractional Differential Equations, Volume 204 of North-Holland Mathematics Studies; Elsevier: Amsterdam, The Netherlands, 2006.

2. Podlubny, I. Fractional Differential Equations, Volume 198 of Mathematics in Science and Engineering; Academic Press: San Diego, CA, USA, 1999.

3. Samko, S.G.; Kilbas, A.A.; Marichev, O.I. Fractional Integrals and Derivatives, Theory and Applications; Gordon and Breach: Zurich, Switzerland, 1993.

4. Henderson, J.; Luca, R. Existence of positive solutions for a singular fractional boundary value problem. Nonlinear Anal. Model. Control 2017, 22, 99-114. [CrossRef]

5. Zhang, X.; Shao, Z.; Zhong, Q.; Zhao, Z. Triple positive solutions for semipositone fractional differential equations $m$-point boundary value problems with singularities and $p$ - $q$-order derivatives. Nonlinear Anal. Model. Control 2018, 23, 889-903. [CrossRef]

6. Zhang, K.; Fu, Z. Solutions for a class of Hadamard fractional boundary value problems with sign-changing nonlinearity. J. Funct. Spaces 2019, 2019, 9046472. [CrossRef]

7. Pu, R.; Zhang, X.; Cui, Y.; Li, P.; Wang, W. Positive solutions for singular semipositone fractional differential equation subject to multipoint boundary conditions. J. Funct. Spaces 2017, 2017, 5892616. [CrossRef]

8. Wei, Z.; Li, Q.; Che, J. Initial value problems for fractional differential equations involving Riemann-Liouville sequential fractional derivative. J. Math. Anal. Appl. 2010, 367, 260-272. [CrossRef]

9. Wei, Z.; Dong, W.; Che, J. Periodic boundary value problems for fractional differential equations involving a Riemann-Liouville fractional derivative. Nonlinear Anal. Theory Methods Appl. 2010, 73, 3232-3238. [CrossRef]

10. Shu, X.; Lai, Y.; Chen, Y. The existence of mild solutions for impulsive fractional partial differential equations. Nonlinear Anal. Theory Methods Appl. 2011, 74, 2003-2011. [CrossRef]

11. Baleanu, D.; Rezapour, S.; Mohammadi, M. Some existence results on nonlinear fractional differential equations. Philos. Trans. A 2013, 371, 20120144. [CrossRef]

12. Samet, B.; Aydi, H. On some inequalities involving Caputo fractional derivatives and applications to special means of real numbers. Mathematics 2018, 6, 193. [CrossRef]

13. Yue, Z.; Zou, Y. New uniqueness results for fractional differential equation with dependence on the first order derivative. Adv. Differ. Equ. 2019, 2019, 38. [CrossRef]

14. Zou, Y.; He, G. The existence of solutions to integral boundary value problems of fractional differential equations at resonance. J. Funct. Spaces 2017, 2017, 2785937. [CrossRef]

15. Zou, Y.; He, G. On the uniqueness of solutions for a class of fractional differential equations. Appl. Math. Lett. 2017, 74, 68-73. [CrossRef]

16. Zou, Y. Positive solutions for a fractional boundary value problem with a perturbation term. J. Funct. Spaces 2018, 2018, 9070247. [CrossRef]

17. Cui, Y. Uniqueness of solution for boundary value problems for fractional differential equations. Appl. Math. Lett. 2016, 51, 48-54. [CrossRef]

18. Cui, Y.; Ma, W.; Sun, Q.; Su, X. New uniqueness results for boundary value problem of fractional differential equation. Nonlinear Anal. Model. Control 2018, 23, 31-39. [CrossRef]

19. Meng, S.; Cui, Y. The extremal solution to conformable fractional differential equations involving integral boundary condition. Mathematics 2019, 7, 186. [CrossRef]

20. Meng, S.; Cui, Y. Multiplicity results to a conformable fractional differential equations involving integral boundary condition. Complexity 2019, 2019, 8402347. [CrossRef] 
21. Cui, Y.; Sun, Q.; Su, X. Monotone iterative technique for nonlinear boundary value problems of fractional order $p \in(2,3]$. Adv. Differ. Equ. 2017, 2017, 248. [CrossRef]

22. Zhang, X.; Wu, J.; Liu, L.; Wu, Y.; Cui, Y. Convergence analysis of iterative scheme and error estimation of positive solution for a fractional differential equation. Math. Model. Anal. 2018, 23, 611-626. [CrossRef]

23. Wu, J.; Zhang, X.; Liu, L.; Wu, Y.; Cui, Y. The convergence analysis and error estimation for unique solution of a $p$-Laplacian fractional differential equation with singular decreasing nonlinearity. Bound. Value Probl. 2018, 2018, 82. [CrossRef]

24. He, J.; Zhang, X.; Liu, L.; Wu, Y.; Cui, Y. Existence and asymptotic analysis of positive solutions for a singular fractional differential equation with nonlocal boundary conditions. Bound. Value Probl. 2018, 2018, 189. [CrossRef]

25. He, J.; Zhang, X.; Liu, L.; Wu, Y.; Cui, Y. A singular fractional Kelvin-Voigt model involving a nonlinear operator and their convergence properties. Bound. Value Probl. 2019, 2019, 112. [CrossRef]

26. Zhong, Q.; Zhang, X.; Lu, X.; Fu, Z. Uniqueness of successive positive solution for nonlocal singular higher-order fractional differential equations involving arbitrary derivatives. J. Funct. Spaces 2018, 2018, 6207682. [CrossRef]

27. Wang, F.; Cui, Y. Unbounded solutions to abstract boundary value problems of fractional differential equations on a half line. Math. Methods Appl. Sci. 2019. [CrossRef]

28. Wang, Y.; Liu, Y.; Cui, Y. Infinitely many solutions for impulsive fractional boundary value problem with p-Laplacian. Bound. Value Probl. 2018, 2018, 94. [CrossRef]

29. Zuo, M.; Hao, X.; Liu, L.; Cui, Y. Existence results for impulsive fractional integro-differential equation of mixed type with constant coefficient and antiperiodic boundary conditions. Bound. Value Probl. 2017, 2017, 161. [CrossRef]

30. Sun, Q.; Ji, H.; Cui, Y. Positive solutions for boundary value problems of fractional differential equation with integral boundary conditions. J. Funct. Spaces 2018, 2018, 6461930. [CrossRef]

31. Sun, Q.; Meng, S.; Cui, Y. Existence results for fractional order differential equation with nonlocal Erdélyi-Kober and generalized Riemann-Liouville type integral boundary conditions at resonance. Adv. Differ. Equ. 2018, 2018, 243. [CrossRef]

32. Ma, W.; Cui, Y. The eigenvalue problem for Caputo type fractional differential equation with Riemann-Stieltjes integral boundary conditions. J. Funct. Spaces 2018, 2018, 2176809. [CrossRef]

33. Zhang, K.; Wang, J.; Ma, W. Solutions for integral boundary value problems of nonlinear Hadamard fractional differential equations. J. Funct. Spaces 2018, 2018, 2193234. [CrossRef]

34. Ma, W.; Meng, S.; Cui, Y. Resonant integral boundary value problems for Caputo fractional differential equations. Math. Probl. Eng. 2018, 2018, 5438592. [CrossRef]

35. Wang, G.; Bai, Z.; Zhang, L. Successive iterations for unique positive solution of a nonlinear fractional q-integral boundary value problem. J. Appl. Anal. Comput. 2019, 9, 1204-1215.

36. Jia, M.; Li, L.; Liu, X.; Song, J.; Bai, Z. A class of nonlocal problems of fractional differential equations with composition of derivative and parameters. Adv. Differ. Equ. 2019, 2019, 280. [CrossRef]

37. Zhang, W.; Bai, Z.; Sun, S. Extremal solutions for some periodic fractional differential equations. Adv. Differ. Equ. 2016, 2016, 179. [CrossRef]

38. Sheng, K.; Zhang, W.; Bai, Z. Positive solutions to fractional boundary-value problems with $p$-Laplacian on time scales. Bound. Value Probl. 2018, 2018, 70. [CrossRef]

39. Dong, X.; Bai, Z.; Zhang, S. Positive solutions to boundary value problems of $p$-Laplacian with fractional derivative. Bound. Value Probl. 2017, 2017, 5. [CrossRef]

40. Tian, Y.; Sun, S.; Bai, Z. Positive solutions of fractional differential equations with $p$-Laplacian. J. Funct. Spaces 2017, 2017, 3187492. [CrossRef]

41. Song, Q.; Bai, Z. Positive solutions of fractional differential equations involving the Riemann-Stieltjes integral boundary condition. Adv. Differ. Equ. 2018, 2018, 183. [CrossRef]

42. He, L.; Dong, X.; Bai, Z.; Chen, B. Solvability of some two-point fractional boundary value problems under barrier strip conditions. J. Funct. Spaces 2017, 2017, 1465623. [CrossRef]

43. Song, Q.; Dong, X.; Bai, Z.; Chen, B. Existence for fractional Dirichlet boundary value problem under barrier strip conditions. J. Nonlinear Sci. Appl. 2017, 10, 3592-3598. [CrossRef]

44. Zhao, Y.; Hou, X.; Sun, Y.; Bai, Z. Solvability for some class of multi-order nonlinear fractional systems. Adv. Differ. Equ. 2019, 2019, 23. [CrossRef] 
45. Bai, Z.; Dong, X.; Yin, C. Existence results for impulsive nonlinear fractional differential equation with mixed boundary conditions. Bound. Value Probl. 2016, 2016, 63. [CrossRef]

46. Zhai, C.; Li, P.; Li, H. Single upper-solution or lower-solution method for Langevin equations with two fractional orders. Adv. Differ. Equ. 2018, 2018, 360. [CrossRef]

47. Fu, Z.; Bai, S.; O’Regan, D.; Xu, J. Nontrivial solutions for an integral boundary value problem involving Riemann-Liouville fractional derivatives. J. Inequal. Appl. 2019, 2019, 104. [CrossRef]

48. Zhang, K.; O'Regan, D.; Xu, J.; Fu, Z. Nontrivial solutions for a higher order nonlinear fractional boundary value problem involving Riemann-Liouville fractional derivatives. J. Funct. Spaces 2019, 2019, 2381530. [CrossRef]

49. Fazli, H.; Nieto, J.J.; Bahrami, F. On the existence and uniqueness results for nonlinear sequential fractional differential equations. Appl. Comput. Math. 2018, 17, 36-47.

50. Bai, Z. On positive solutions of a nonlocal fractional boundary value problem. Nonlinear Anal. 2010, 72, 916-924. [CrossRef]

51. Jiang, J.; O’Regan, D.; Xu, J.; Cui, Y. Positive solutions for a Hadamard fractional $p$-Laplacian three-point boundary value problem. Mathematics 2019, 7, 439. [CrossRef]

52. Jiang, J.; O'Regan, D.; Xu, J.; Fu, Z. Positive solutions for a system of nonlinear Hadamard fractional differential equations involving coupled integral boundary conditions. J. Inequal. Appl. 2019, $2019,204$. [CrossRef]

53. Ahmad, B.; Ntouyas, S.K.; Alsaedi, A. On a coupled system of fractional differential equations with coupled nonlocal and integral boundary conditions. Chaos Solitons Fractals 2016, 83, 234-241. [CrossRef]

54. Wang, Y.; Liu, L.; Zhang, X.; Wu, Y. Positive solutions of an abstract fractional semipositone differential system model for bioprocesses of HIV infection. Appl. Math. Comput. 2015, 258, 312-324. [CrossRef]

55. Aljoudi, S.; Ahmad, B.; Nieto, J.J.; Alsaedi, A. A coupled system of Hadamard type sequential fractional differential equations with coupled strip conditions. Chaos Solitons Fractals 2016, 91, 39-46. [CrossRef]

56. Henderson, J.; Luca, R.; Tudorache, A. On a system of fractional differential equations with coupled integral boundary conditions. Fract. Calc. Appl. Anal. 2015, 18, 361-386. [CrossRef]

57. Ahmad, B.; Luca, R. Existence of solutions for a system of fractional differential equations with coupled nonlocal boundary conditions. Fract. Calc. Appl. Anal. 2018, 21, 423-441. [CrossRef]

58. Henderson, J.; Luca, R. Positive solutions for a system of coupled fractional boundary value problems. Lith. Math. J. 2018, 58, 15-32. [CrossRef]

59. Ahmad, B.; Nieto, J.J. Existence results for a coupled system of nonlinear fractional differential equations with three-point boundary conditions. Comput. Math. Appl. 2009, 58, 1838-1843. [CrossRef]

60. Ali, A.; Shah, K.; Jarad, F.; Gupta, V.; Abdeljawad, T. Existence and stability analysis to a coupled system of implicit type impulsive boundary value problems of fractional-order differential equations. Adv. Differ. Equ. 2019, 2019, 101. [CrossRef]

61. Mahmudov, N.I.; Bawaneh, S.; Al-Khateeb, A. On a coupled system of fractional differential equations with four point integral boundary conditions. Mathematics 2019, 7, 279. [CrossRef]

62. Zhao, Y.; Chen, H.; Xu, C. Nontrivial solutions for impulsive fractional differential equations via Morse theory. Appl. Math. Comput. 2017, 307, 170-179. [CrossRef]

63. Zhang, X.; Liu, L.; Wu, Y.; Zou, Y. Existence and uniqueness of solutions for systems of fractional differential equations with Riemann-Stieltjes integral boundary condition. Adv. Differ. Equ. 2018, 2018, 204. [CrossRef]

64. Zhang, X.; Liu, L.; Zou, Y. Fixed-point theorems for systems of operator equations and their applications to the fractional differential equations. J. Funct. Spaces 2018, 2018, 7469868. [CrossRef]

65. Hao, X.; Wang, H.; Liu, L.; Cui, Y. Positive solutions for a system of nonlinear fractional nonlocal boundary value problems with parameters and p-Laplacian operator. Bound. Value Probl. 2017, 2017, 182. [CrossRef]

66. Qi, T.; Liu, Y.; Zou, Y. Existence result for a class of coupled fractional differential systems with integral boundary value conditions. J. Nonlinear Sci. Appl. 2017, 10, 4034-4045. [CrossRef]

67. Qi, T.; Liu, Y.; Cui, Y. Existence of solutions for a class of coupled fractional differential systems with nonlocal boundary conditions. J. Funct. Spaces 2017, 2017, 6703860. [CrossRef]

68. Zhang, Y. Existence results for a coupled system of nonlinear fractional multi-point boundary value problems at resonance. J. Inequal. Appl. 2018, 2018, 198. [CrossRef] [PubMed]

69. Li, H.; Zhang, J. Positive solutions for a system of fractional differential equations with two parameters. J. Funct. Spaces 2018, 2018, 1462505. [CrossRef] 
70. Zhai, C.; Wang, W.; Li, H. A uniqueness method to a new Hadamard fractional differential system with four-point boundary conditions. J. Inequal. Appl. 2018, 2018, 207. [CrossRef]

71. Cheng, W.; Xu, J.; Cui, Y.; Ge, Q. Positive solutions for a class of fractional difference systems with coupled boundary conditions. Adv. Differ. Equ. 2019, 2019, 249. [CrossRef]

72. Cheng, W.; Xu, J.; Cui, Y. Positive solutions for a system of nonlinear semipositone fractional $q$-difference equations with $q$-integral boundary conditions. J. Nonlinear Sci. Appl. 2017, 10, 4430-4440. [CrossRef]

73. Xu, J.; Goodrich, C.S.; Cui, Y. Positive solutions for a system of first-order discrete fractional boundary value problems with semipositone nonlinearities. Rev. R. Acad. Cienc. Exactas Fís. Nat. Ser. A Mat. 2019, 113, 1343-1358. [CrossRef]

74. Qiu, X.; Xu, J.; O’Regan, D.; Cui, Y. Positive solutions for a system of nonlinear semipositone boundary value problems with Riemann-Liouville fractional derivatives. J. Funct. Spaces 2018, 2018, 7351653. [CrossRef]

75. Chen, C.; Xu, J.; O’Regan, D.; Fu, Z. Positive solutions for a system of semipositone fractional difference boundary value problems. J. Funct. Spaces 2018, 2018, 6835028. [CrossRef]

76. Wang, F.; Cui, Y.; Zhou, H. Solvability for an infinite system of fractional order boundary value problems. Ann. Funct. Anal. 2019, 10, 395-411. [CrossRef]

77. Wang, F.; Cui, Y. Positive solutions for an infinite system of fractional order boundary value problems. Adv. Differ. Equ. 2019, 2019, 169. [CrossRef]

78. Riaz, U.; Zada, A.; Ali, Z.; Ahmad, M.; Xu, J.; Fu, Z. Analysis of nonlinear coupled systems of impulsive fractional differential equations with Hadamard derivatives. Math. Probl. Eng. 2019, 2019, 5093572. [CrossRef]

79. Riaz, U.; Zada, A.; Ali, Z.; Cui, Y.; Xu, J. Analysis of coupled systems of implicit impulsive fractional differential equations involving Hadamard derivatives. Adv. Differ. Equ. 2019, 2019, 226. [CrossRef]

80. Aris, R. Introduction to the Analysis of Chemical Reactors; Prentice Hall: Englewood Cliffs, NJ, USA, 1965.

81. Guo, D.; Lakshmikantham, V. Nonlinear Problems in Abstract Cones; Academic Press: Orlando, FL, USA, 1988.

82. Yang, Z.; Zhang, Z. Positive solutions for a system of nonlinear singular Hammerstein integral equations via nonnegative matrices and applications. Positivity 2012, 16, 783-800. [CrossRef]

83. Abraham, B.; Plemmons, R.J. Nonnegative Matrices in the Mathematical Sciences; Academic Press: New York, NY, USA, 1979.

(C) 2019 by the authors. Licensee MDPI, Basel, Switzerland. This article is an open access article distributed under the terms and conditions of the Creative Commons Attribution (CC BY) license (http:// creativecommons.org/licenses/by/4.0/). 